\title{
The predictive effect of the platelet-to-lymphocyte ratio (PLR) and the neutrophil-to-lymphocyte ratio (NLR) on the risk of death in patients with severe fever with thrombocytopenia syndrome (SFTS): a multi-center study in China
}

\author{
Xiankun Wang ${ }^{1 \#}$, Ling Lin ${ }^{2 \#}$, Zhenghua Zhao ${ }^{3 \#}$, Wei Zhou ${ }^{4 \#}$, Zirou Ge ${ }^{1}$, Yi Shen ${ }^{5}$, Lin Wang ${ }^{1}$, Wei Zhang ${ }^{1}$, \\ Rui Song ${ }^{1}$, Di $\operatorname{Tian}^{1}$, Jing Wen ${ }^{1}$, Shuping Cui ${ }^{1}$, Xiaoli Yu ${ }^{5}$, Yang Feng ${ }^{3}$, Yuanni Liu ${ }^{2}$, Chunqian Qiang ${ }^{2}$, \\ Jianping Duan ${ }^{6}$, Yanli Ma ${ }^{6}$, Xingwang Li ${ }^{1}$, Tianli Fan ${ }^{6}$, Yongxiang Zhao ${ }^{5}$, Zhihai Chen ${ }^{1}$ \\ ${ }^{1}$ Center of Infectious Diseases, Beijing Ditan Hospital, Capital Medical University. Beijing, China; ${ }^{2}$ Department of Infectious Diseases, Yantai \\ City Hospital for Infectious Disease, Yantai, China; ${ }^{3}$ Department of Infectious Diseases, Taian City Central Hospital, Taian, China; ${ }^{4}$ Department \\ of Infectious Diseases, Dalian sixth people's hospital, Dalian, China; ${ }^{5}$ Department of Infectious Diseases, Dandong Infectious Disease Hospital, \\ Dandong, China; ${ }^{\circ}$ Department of Infectious Diseases, Qing Dao No. 6 People's Hospital, Qingdao, China \\ Contributions: (I) Conception and design: X Wang, Z Chen; (II) Administrative support: Z Chen, T Fan, Y Zhao; (III) Provision of study materials or \\ patients: L Lin, Z Zhao, W Zhou, Y Shen, Y Zhao; (IV) Collection and assembly of data: Z Ge, L Wang, W Zhang, R Song, D Tian, J Wen, S Cui, \\ X Ren, X Yu, Y Feng, Y Liu, C Qiang, J Duan, Y Ma, X Li; (V) Data analysis and interpretation: X Wang, Z Ge, L Wang; (VI) Manuscript writing: \\ All authors; (VII) Final approval of manuscript: All authors. \\ "These authors contributed equally to this work. \\ Correspondence to: Zhihai Chen. Capital Medical University, 8 Jingshun East Street, Chaoyang District, Beijing, China. Email: chenzhihai0001@126.com; \\ Tianli Fan. Qing Dao No. 6 People’s Hospital, No. 9 Fushun Road, Sifang District, Qingdao, China. Email: 1566668786@163.com; \\ Yongxiang Zhao. Dandong Infectious Disease Hospital, No. 38, Taoyuan Street, Zhenxing District, Dandong, China. Email: ddcrbyy@163.com.
}

Background: Severe fever with thrombocytopenia syndrome is caused by infection with the severe fever with thrombocytopenia syndrome virus.

Methods: Between April 2011 and December 2019, data on consecutive patients who were diagnosed with severe fever with thrombocytopenia syndrome were prospectively collected from five medical centers in China. The score of the death risk model was correlated with the platelet-to-lymphocyte ratio and the neutrophil-to-lymphocyte ratio. Multivariable Cox analyses were used to identify the independent factors associated with mortality.

Results: During the study period, 763 patients were diagnosed with severe fever with thrombocytopenia syndrome; 415 of these patients were enrolled in our study. We found that the neutrophil-to-lymphocyte ratio of the group that died was significantly higher on admission $(\mathrm{P}=0.007)$ than that of the group that survived, and the neutrophil-to-lymphocyte ratio showed a positive correlation with the score of the death risk model. Multivariate Cox regression suggested that a neutrophil-to-lymphocyte ratio greater than 5.4 was an independent risk factor for survival time $(\mathrm{HR}=6.767, \mathrm{P}=0.011)$. Platelet-to-lymphocyte ratio did not show a special role in this study.

Conclusions: A neutrophil-to-lymphocyte ratio greater than 5.4 can increase the risk of death and decrease the survival time of patients. In summary, the neutrophil-to-lymphocyte ratio provides a supplementary means for effectively managing severe fever with thrombocytopenia syndrome (SFTS).

Keywords: Severe fever with thrombocytopenia syndrome (SFTS); neutrophil-to-lymphocyte ratio; platelet-tolymphocyte ratio

Submitted Jun 16, 2020. Accepted for publication Oct 30, 2020.

doi: $10.21037 /$ atm-20-4736

View this article at: http://dx.doi.org/10.21037/atm-20-4736 


\section{Introduction}

Severe fever with thrombocytopenia syndrome (SFTS) is a severe hemorrhagic fever disease caused by the severe fever with thrombocytopenia syndrome virus (SFTSV) (1). According to the latest virus classification issued by the International Virus Classification Committee in 2019, SFTSV belongs to the Phlebovirus genus in the Bunyaviridae family (2). It is a tick-borne disease. Previous research has established that a number of different kinds of animals serve as reservoir hosts and facilitate its spread (3). The disease can be transmitted from person to person through the bodily fluids of infected individuals (4). It is fairly broadly distributed in China (5), and it has also been reported in other parts of Asia, including South Korea and Japan $(6,7)$. Other newly emerged phleboviruses were isolated from patients in the USA in 2012, including the Heartland virus, which is genetically similar to SFTSV (8). Patients infected with SFTSV may develop such clinical manifestations as fever, thrombocytopenia, leukopenia, gastrointestinal symptoms, mental symptoms, diffuse intravascular coagulation (DIC), and even multiple organ dysfunction (9).

Studies have revealed that the pathogenesis of SFTS is closely related to an abnormal immune response $(10,11)$. Increasing evidence suggests that a patient's platelet-tolymphocyte ratio (PLR) and neutrophil-to-lymphocyte ratio (NLR) serve as inflammatory markers; both have been used as prognostic factors or progress indicators for various diseases $(12,13)$. Current research has suggested that elevated NLR increased the risk of all-cause cardiovascular death in patients (12). Diabetic status was also associated with a significant increase in NLR (14). Previous research showed that tumors were associated with chronic inflammation of the body and the importance of neutrophils in cancer has become increasingly apparent $(15,16)$. The progression-free survival of breast cancer patients with a low NLR at baseline was significantly better than that of patients with a high NLR at baseline (17). Previous studies also revealed the NLR was correlated with the risk of death in patients with severe exacerbation of chronic hepatitis B (18). As for the PLR, in patients with Crimean-Congo hemorrhagic fever, there was a strong relationship between the severity of the disease and the PLR (13).

SFTS is a newly emerging infectious disease with a high mortality rate (11.2-30\%) (19), and the number of cases is increasing each year. For these reasons, the ability to predict the severity of the disease is of particular importance. The NLR and PLR are commonly assessed and it is easy to obtain these indicators from the laboratory in clinical work. However, there is currently no research being done on the role NLR and PLR play in SFTS. For this reason, we intend to study the role of NLR and PLR in predicting the severity of this disease. We present the following article in accordance with the STROBE reporting checklist (available at http://dx.doi.org/10.21037/ atm-20-4736).

\section{Methods}

\section{Study design}

For this prospective study, we recruited SFTS patients from April 2011 to December 2019 from five medical centers located in northeastern and central China: Beijing Ditan Hospital, Dandong Infectious Diseases Hospital, Tai'an City Center Hospital, Qingdao Sixth People's Hospital, and Yantai Infectious Diseases Hospital. Patients had to meet all of the following conditions for inclusion: (I) maintained a fever $\left(\mathrm{T}>37.5^{\circ} \mathrm{C}\right)$ for over $24 \mathrm{~h}$; (II) had reduced platelet count in peripheral blood in laboratory tests $\left(\mathrm{PLT}<100 \times 10^{9} / \mathrm{L}\right)$; (III) real-time fluorescent polymerase chain reaction (RT-PCR) was positive for SFTS virus RNA and/or SFTSV IgM was positive in the patient's blood. Patients were excluded if they met any of the following conditions: (I) had previous autoimmune diseases such as systemic lupus erythematosus (SLE); (II) had previous blood system diseases such as aplastic diseases, leukemia, and idiopathic thrombocytopenic purpura; (III) had previous liver disease such as acute and chronic viral hepatitis, chronic alcoholic liver disease; (IV) had an infusion of blood product platelet transfusions or treatment with stimulating factors such as granulocyte stimulating factor; (V) had previous cardiovascular and cerebrovascular diseases such as hypertension, coronary heart disease, cerebral hemorrhage, and cerebral infarction; (VI) had previous metabolic diseases such as diabetes and hyperthyroidism.

This study was approved by the Local Ethics Committee of the Leading Center of Beijing Ditan Hospital, Capital Medical University (No. 2014-003) and was in line with the principles of the Helsinki Declaration (as revised in 2013). Informed consent was obtained from all patients or their immediate family members for sample and data collection and use in research. 


\section{Data collection}

We collected patient demographic data such as gender and age, the rate of occurrence of neurological symptoms, and the first laboratory indicators within 7 days of symptom onset and last laboratory indicators in the blood of the patient during hospitalization such as white blood cell count (WBC), neutrophil count (NEU), platelet count (PLT), lymphocyte count (LYM), monocytes (MONO), eosinophilic granular cell (EO), basophil (BASO), hemoglobin $(\mathrm{Hb})$, alanine aminotransferase (ALT), aspartate aminotransferase (AST), prothrombin time (PT), activated partial thromboplastin time (APTT), creatine kinase (CK), lactic dehydrogenase (LDH), gamma glutamine transferase (GGT), alkaline phosphatase (ALP), choline esterase (CHE), adenosine deaminase (ADA), total bile acid (TBA), creatine kinase (CK), uric acid (UA), blood creatinine (sCr), blood urea nitrogen (BNU), NLR, and PLR.

Deaths are defined as patients who died during the course of the disease. The recovery period of the disease was defined as the time frame in which the patient's temperature gradually returned to normal, the symptoms and signs gradually improved, and the patient reached the level at which he or she could be discharged.

\section{Statistical analysis}

Data analysis was performed using SPSS24.0 software (IBM, Armonk, NY, USA). The measurement data with a normal distribution were expressed as mean \pm standard deviation, and the comparison between groups used independent sample $t$-test. The measurement data with a skewed distribution were expressed as medians with interquartile ranges and the comparison between the two groups used Mann-Whitney U tests. Chi-square tests were used for comparing differences in proportion between groups. Univariable and multivariate Cox regression analyses were used to identify dependent and independent risk factors that affected patient survival time.

\section{Results}

\section{Demographic characteristics of the patients}

A total of 763 laboratory-confirmed SFTS patients were enrolled in our study between April 2011 and December 2019. After excluding cases through the exclusion criteria, 415 patients were finally included in our study. Among the included cases, 352 survived and 63 died. There were
241 male patients and 174 female patients. The mean age of all patients was 60.16 years. Notably, the mean age and the occurrence of neurologic symptoms in patients who died were significantly higher than in those who survived (67.32 vs. 58.88; $\mathrm{P}<0.001$ ), and there was also a significant difference in the gender distribution between the death and survival groups. The demographic characteristics of the patients are presented in Table 1 .

\section{Comparison of laboratory parameters in surviving and fatal SFTS cases}

From our data, we found that the neutrophil ratio (NEU\%), NEU, BASO, Hb, ALT, AST, GGT, ALP, ADA, TBA, $\mathrm{LDH}, \mathrm{sCr}, \mathrm{UA}, \mathrm{BUN}, \mathrm{PT}$, APTT, and CK in the group that died were significantly higher than those in the group that survived. We also found that the LYM and the lymphocyte ratio (LYM\%) in the group that died were lower than those in the group that survived. There was no significant difference between the two groups in the PLR. The clinical characteristics of the patients are presented in Table 1.

\section{Comparison of the NLR and the PLR of patients with SFTSV at different stages of the disease}

We collected the first and last blood tests of patients in the group that survived and the group that died during their hospital stay and compared the changes in their NLR and PLR. We found that the NLR at the time of discharge in the group that survived was significantly lower than the NLR had been at the time of admission (Figure 1A), with statistical significance $(\mathrm{P}<0.001)$. The PLR at the time of discharge in the group that survived was significantly higher than it had been at admission (Figure $1 B$ ), with statistical significance $(\mathrm{P}<0.001)$. For the group that died, we found that the NLR at the time of admission was lower than the NLR at the time of death (Figure 1C) $(\mathrm{P}<0.001)$. In the group that died, the PLR at the time of admission was significantly higher than the PLR at the time of death (Figure 1D), with statistical significance $(\mathrm{P}<0.001)$. Regarding the NLR of the group that survived and the group that died, we found that the NLR at the time of discharge for the group that survived was lower than the NLR at the time of death for the group that died (Figure 1E), with statistical significance $(\mathrm{P}<0.001)$. The PLR at the time of discharge for the group that survived was higher than the PLR at the time of death for the group that died (Figure $1 F$ ), with statistical significance 
Table 1 Comparison of gender, age, neurologic symptoms (NS), and laboratory parameters in the survival and fatal groups

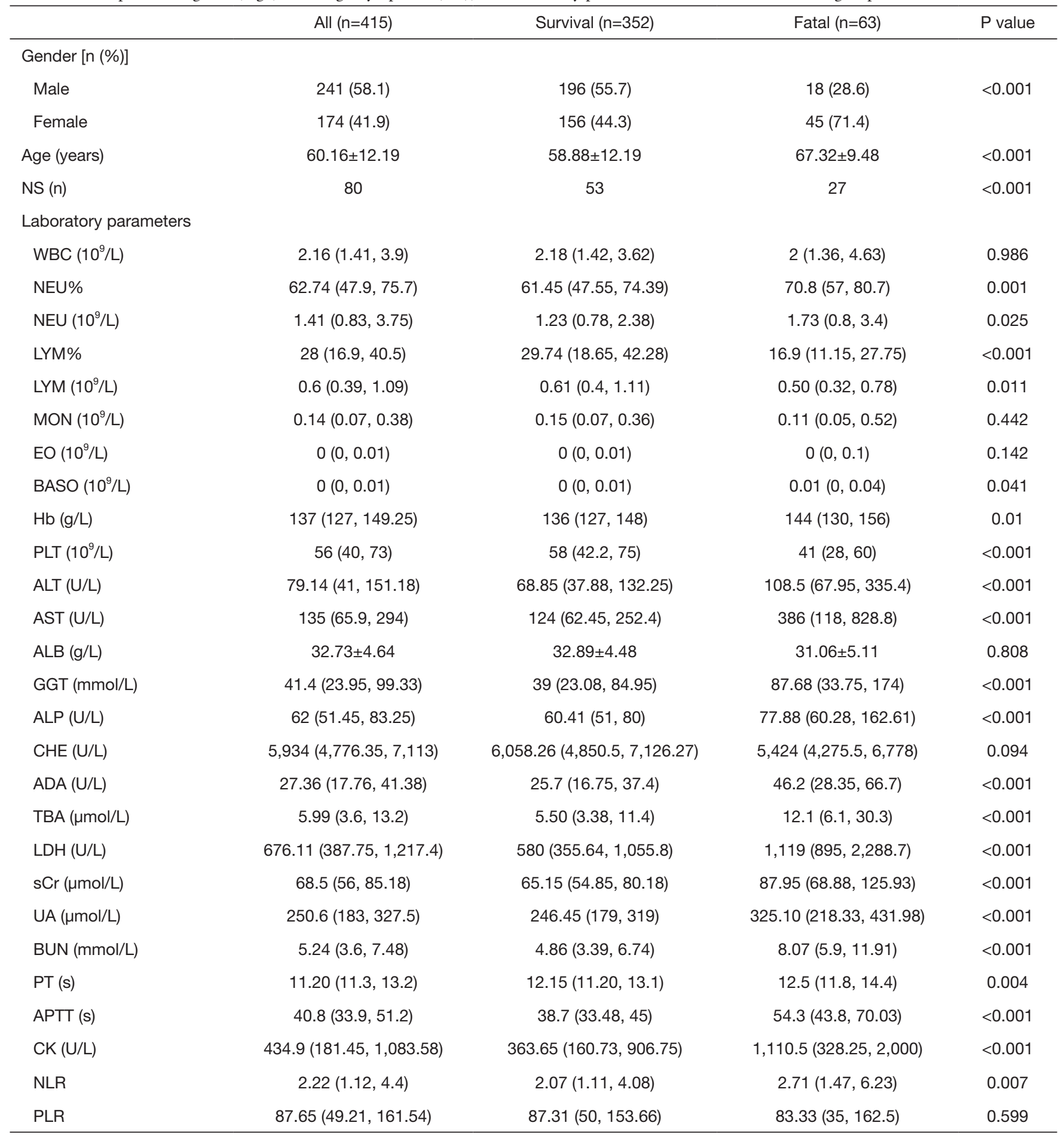

WBC, white blood cell; NEU\%, neutrophil percentage; NEU, neutrophil; LYM\%, lymphocyte percentage; LYM, lymphocyte; MON, monocyte; EO, eosinophilic cell; BASO, basophil; Hb, hemoglobin; PLT, platelet count; LDH, lactate dehydrogenase; CK, creatinine kinase; BUN, Blood urea nitrogen; sCr, serum creatinine; PT, prothrombin time; PTA, prothrombin activity; APTT, activated partial thromboplastin time; ALP, alkaline phosphatase; ALT, alanine aminotransferase; AST, aspartate aminotransferase; TB, total bilirubin; ALB, albumin; ADA, adenosine deaminase; UA, uric acid; CHE, choline esterase; GGT, Gamma glutamine transferase; NS, neurologic symptoms; NLR, neutrophil-to-lymphocyte ratio; PLR, platelet-to-lymphocyte ratio. 

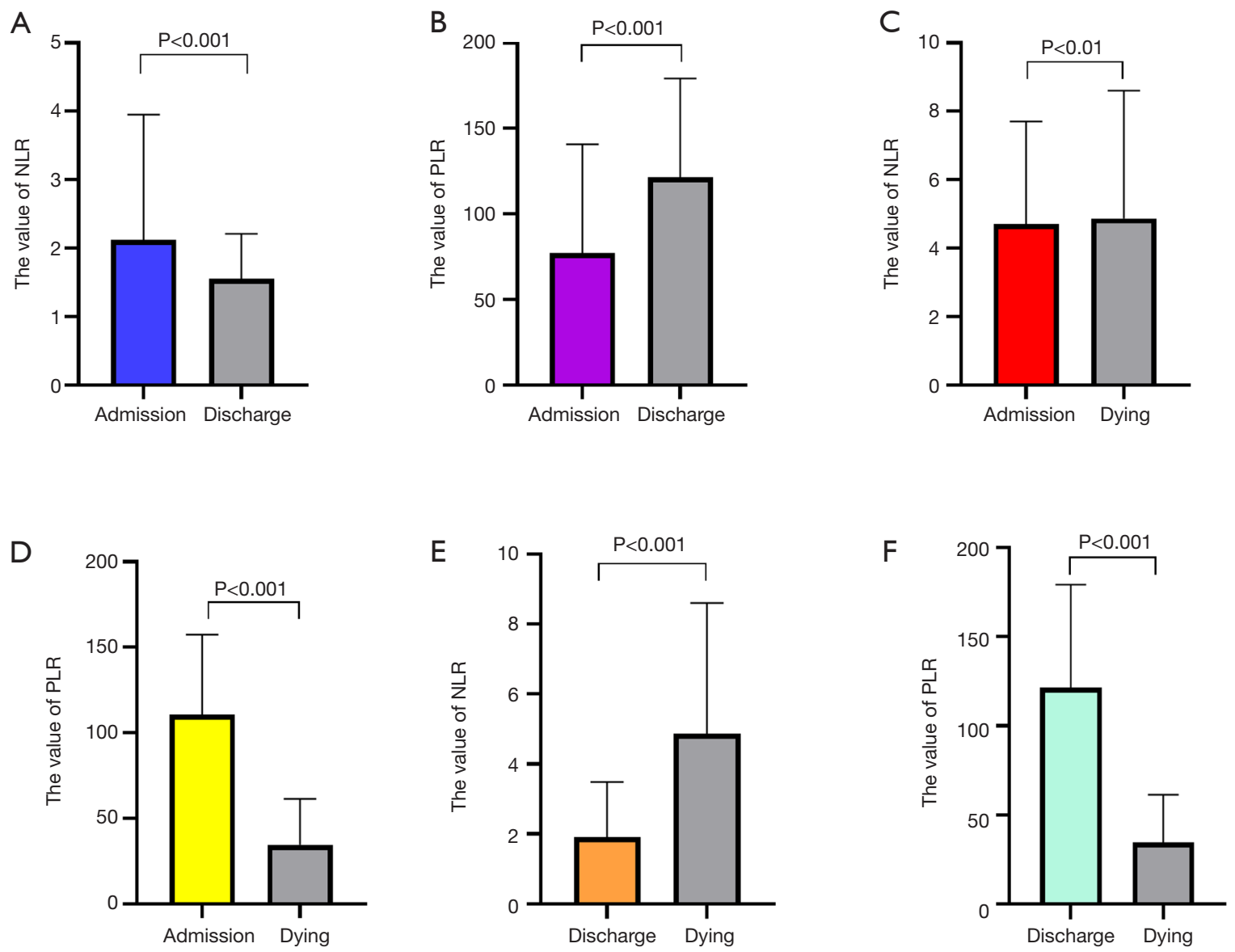

Figure 1 Comparison of NLR and PLR of patients with SFTS at different stages of the disease. NLR, neutrophil-to-lymphocyte ratio; PLR, platelet-to-lymphocyte ratio; SFTS, severe fever with thrombocytopenia syndrome.

$(\mathrm{P}<0.001)$

\section{Correlation between NLR / PLR and score from the clinical model predicting mortality}

Using the clinical model predicting mortality constructed by Li (20), we put the six indicators of age, neurological symptoms, LDH, AST, BUN, and NEU\% within 6 days of the onset of symptoms into the model and obtained the scores of the group that survived (Figure $2 A$ ), the group that died (Figure 2B), and the overall situation (Figure 2C). We correlated the total score and the corresponding NLR (Figure $3 A$ ) and PLR (Figure $3 B$ ) values and found that the NLR was highly positively correlated with the score $(\mathrm{r}=0.399, \mathrm{P}<0.001)$, while the PLR was not correlated with the score $(\mathrm{r}=0.023, \mathrm{P}=0.735)$.

\section{Risk factors for fatal outcomes}

Based on our assignment of the corresponding indicators (Table 2), we performed Cox regression analyses. Univariable Cox regression analysis revealed that older age, decreased percentage of lymphocytes, incidence of neurologic symptoms, elevated percentage of neutrophils, and elevated levels of CK, LDH, ALT, AST, BUN, sCr, ALP, ADA, TBA, GGT, NLR, and APTT were risk factors for fatal outcomes. The above variables were used for multivariable Cox regression analysis. The results indicated that NLR >5.4 and an elevated concentration of serum UA were independent risk factors for fatal outcomes. The detailed results of the multivariable analysis are shown in Table 3. The survival curve of the NLR is shown in Figure 4. 

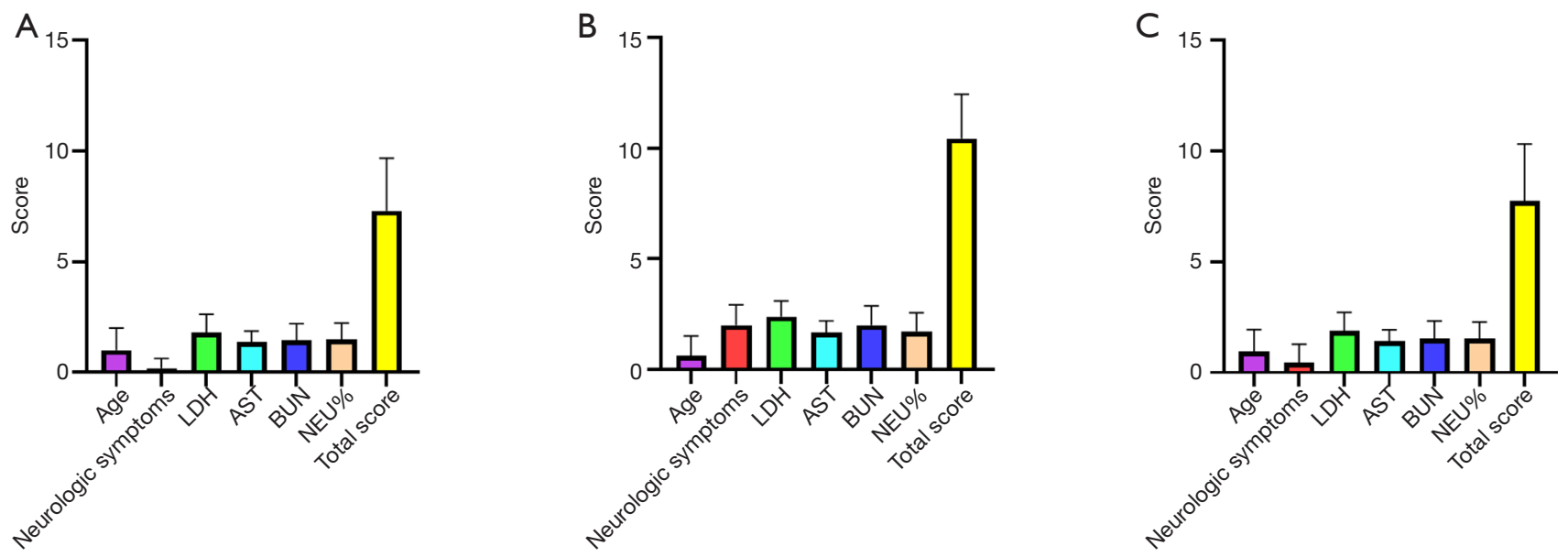

Figure 2 Model score of the group that survived and group that died and overall situation. LDH, lactate dehydrogenase; AST, aspartate aminotransferase; NEU (\%), neutrophil percentage; BUN, blood urea nitrogen.
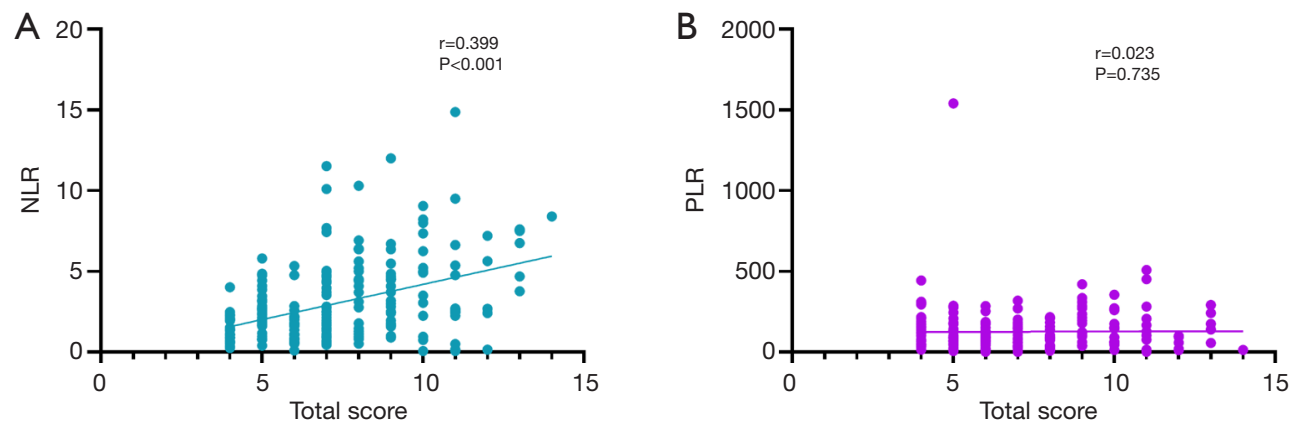

Figure 3 Scatter plot of correlation between NLR/PLR and total score. NLR, neutrophil-to-lymphocyte ratio; PLR, platelet-to-lymphocyte ratio.

\section{Discussion}

SFTS is a newly emerging infectious disease with a high mortality rate. In addition, the number of cases increases each year. In this study, we prospectively analyzed a large cohort of SFTS patients that was well-characterized and laboratory-confirmed between April 2011 and December 2019.

After implementation of our inclusion and exclusion criteria, our cohort showed differences between the group that died and the group that survived. Regarding gender, we found that there were significant differences between the group that died and the group that survived. The number of women in the group that died was higher than the number of men. This result was different from Li's (20) research involving a larger SFST cohort, which showed that, based on gender, there was no difference between the group that died and the group that survived. We speculated that this was caused by the exclusion criteria as we did not include patients with underlying diseases and patients who received treatment such as blood products. The neurological symptoms and the number of elderly patients in the group that died were significantly higher than in the group that survived, which was consistent with the results of most studies (21). In terms of laboratory indicators, NEU\%, NEU, Hb, ALT, AST, GGT, ALP, ADA, TBA, LDH, sCr, UA, BUN, PT, APTT, and CK levels in the group that died were significantly higher than those in the group that survived. It was worth noting that the counts of BASO in the group that survived were significantly higher than those in the group that died. There is currently no corresponding study that explores the relationship between SFTS and BASO. The role of basophils is often 
Table 2 Variables and assignments of SFTS risk factors analysis

\begin{tabular}{|c|c|c|}
\hline \multirow{2}{*}{$\begin{array}{l}\text { Variable name } \\
\text { Sex }\end{array}$} & \multicolumn{2}{|c|}{ Assignment description } \\
\hline & Male $=1$ & Female $=0$ \\
\hline Age (years) & $\geq 65=1$ & $<65=0$ \\
\hline WBC $\left(\times 10^{9} / L\right)$ & $>10=1$ & $\leq 10=0$ \\
\hline NE\% & $>70 \%=1$ & $\leq 70 \%=0$ \\
\hline $\mathrm{NE}\left(10^{9} / \mathrm{L}\right)$ & $>7.5=1$ & $\leq 7.5=0$ \\
\hline LYM\% & $<17=1$ & $\geq 17=0$ \\
\hline $\operatorname{LYM}\left(10^{9} / \mathrm{L}\right)$ & $<0.8=1$ & $\geq 0.8=0$ \\
\hline MON $\left(10^{9} / L\right)$ & $<0.3=1$ & $\geq 0.3=0$ \\
\hline EO $\left(10^{9} / \mathrm{L}\right)$ & $<0.05=1$ & $\geq 0.05=0$ \\
\hline BASO $\left(10^{9} / \mathrm{L}\right)$ & $<0.05=1$ & $\geq 0.05=0$ \\
\hline $\mathrm{Hb}(\mathrm{g} / \mathrm{L})$ & $<110=1$ & $\geq 110=0$ \\
\hline PLT $\left(10^{9} / \mathrm{L}\right)$ & $<100=1$ & $\geq 100=0$ \\
\hline LDH (U/L) & $>215=1$ & $\leq 215=0$ \\
\hline CK (U/L) & $>198=1$ & $\leq 198=0$ \\
\hline BUN (mmol/L) & $>7=1$ & $\leq 7=0$ \\
\hline $\mathrm{sCr}(\mu \mathrm{mol} / \mathrm{L})$ & $>100=1$ & $\leq 100=0$ \\
\hline $\mathrm{UA}(\mu \mathrm{mol} / \mathrm{L})$ & $>420=1$ & $\leq 420=0$ \\
\hline PT (s) & $>17=1$ & $\leq 17=0$ \\
\hline APTT (s) & $>47=1$ & $\leq 47=0$ \\
\hline ALT (U/L) & $>120=1$ & $\leq 120=0$ \\
\hline AST (U/L) & $>120=1$ & $\leq 120=0$ \\
\hline ALB (g/L) & $<25=1$ & $\geq 25=0$ \\
\hline GGT (U/L) & $>50=1$ & $\leq 50=0$ \\
\hline ALP (U/L) & $>125=1$ & $\leq 125=0$ \\
\hline ADA (U/L) & $>22=1$ & $\leq 22=0$ \\
\hline TBA $(\mu \mathrm{mol} / \mathrm{L})$ & $>10=1$ & $\leq 10=0$ \\
\hline NLR & $>5.4=1$ & $\leq 5.4=0$ \\
\hline NS & Existance $=1$ & None $=0$ \\
\hline PLR & $<60=1$ & $\geq 60=0$ \\
\hline
\end{tabular}

WBC, white blood cell; NEU\%, neutrophil percentage; NEU, neutrophil; LYM\%, lymphocyte percentage; LYM, lymphocyte; MON, monocyte; EO, eosinophilic cell; BASO, basophil; Hb, hemoglobin; PLT, platelet count; LDH, lactate dehydrogenase; $\mathrm{CK}$, creatinine kinase; BUN, Blood urea nitrogen; sCr, serum creatinine; PT, prothrombin time; PTA, prothrombin activity; APTT, activated partial thromboplastin time; ALP, alkaline phosphatase; ALT, alanine aminotransferase; AST, aspartate aminotransferase; TB, total bilirubin; ALB, albumin; ADA, adenosine deaminase; UA, uric acid; CHE, choline esterase; NS, neurologic symptoms; NLR, neutrophil-to-lymphocyte ratio; PLR, platelet-to-lymphocyte ratio. overlooked in clinical work, but one study has shown that basophils have antiparasitic and immunomodulatory effects (22). A study by Yoshikawa (23) has shown that BASO levels are regulated by IL-3, therefore, we can do further research in this field in the future.

As an indicator of inflammation, from our research, PLR also showed an effect on SFTS. We found that the NLR of the group that died was significantly higher than that of the group the survived in the early days after admission. The NLR at the time of death for the group that died was also significantly higher than that of group that survived at the last measurement taken during hospitalization. Together, these data suggest that the inflammatory storm might be significantly stronger in the group that died than in the group that survived. Our research showed that the NLR of patients in the recovery phase was lower than in the acute phase, which was consistent with the patients' improvement. In line with our expectations, the NLR of patients who subsequently died was statistically lower at the time of admission than at the time of death, which was consistent with the patients' deterioration. We used a death score model to evaluate patients in our cohort and found that the NLR was positively correlated with the score, suggesting that an increase in NLR might be related to patient death. However, unlike the relatively low NLR $(14,24,25)$, which predicted the poor prognosis of solid tumors and chronic viral infection, we used Cox regression analysis, which suggested that an NLR greater than 5.4 was an independent risk factor for SFTS death, which was not revealed in previous studies. We considered that this might be related to the mechanism of tumorigenesis involving chronic infection rather than acute infection. Liu's (26) research provided evidence for this guess. We then added the survival time to the study. The mechanism by which inflammation led to worse outcomes was not clear. Neutrophilia was considered to occur as an inflammatory response and might lead to suppression of the cytolytic activity of immune cells such as lymphocytes, natural killer cells, and activated T cells (27). Another study (14) showed the NLR because of the shorter lifespan of neutrophils than lymphocytes; however, neutrophils could be actively recruited during acute phase reactions and act as a marker of disease severity and tissue inflammation. Recent evidence has suggested that thrombocytopenia and neutropenia are related to the release of neutrophil extracellular traps (NETs) (28). NETs consist of uncoiled chromatin DNA, histones, and granule proteins, such as myeloperoxidase, neutrophil elastase, and lactoferrin (29). NETs can prevent viral 
Table 3 Univariable and multivariable Cox regression analyses of features associated with fatal outcomes in patients with SFTS

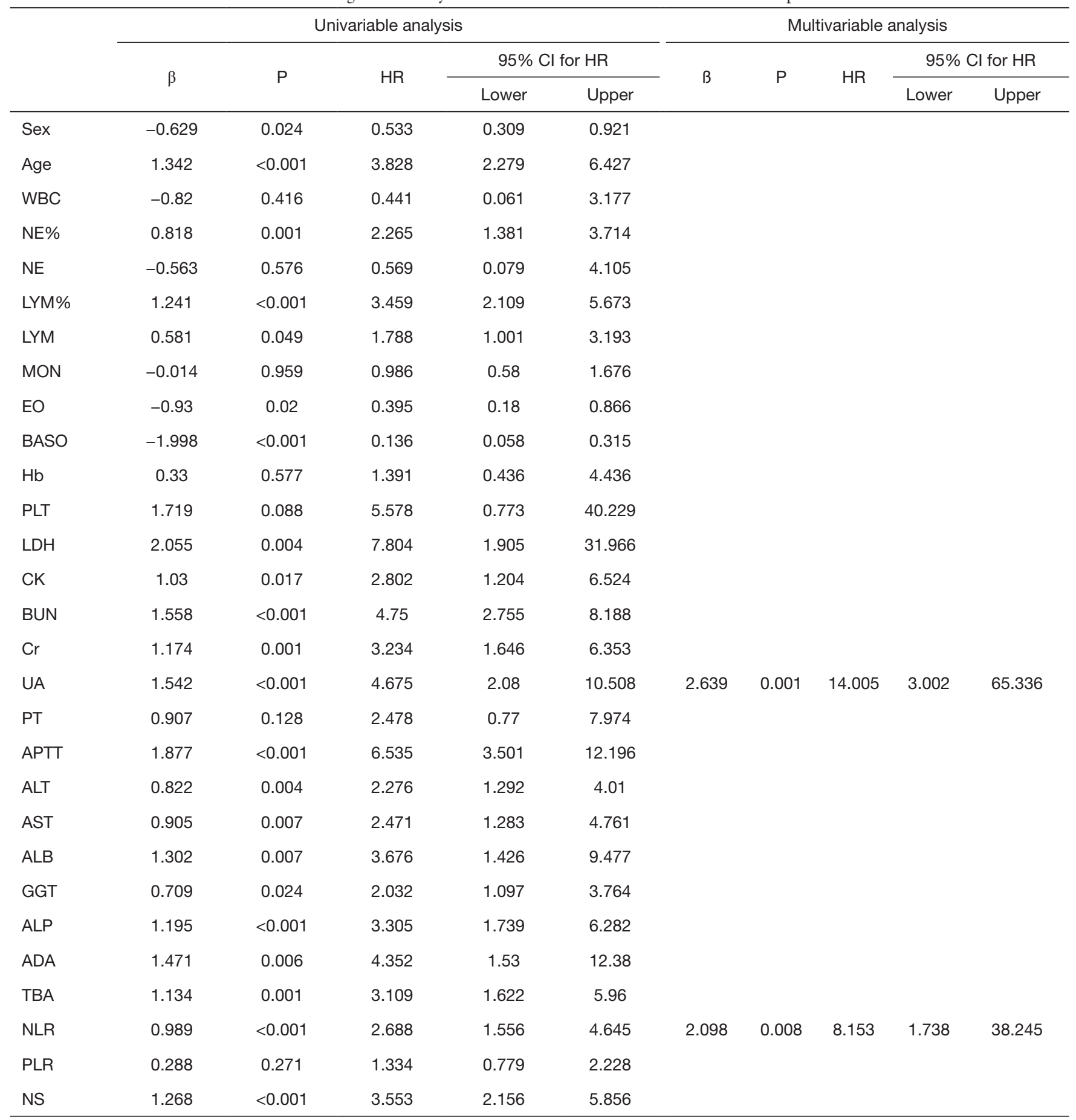

WBC, white blood cell; NEU\%, neutrophil percentage; NEU, neutrophil; LYM\%, lymphocyte percentage; LYM, lymphocyte; MON, monocyte; EO, eosinophilic cell; BASO, basophil; Hb, hemoglobin; PLT, platelet count; LDH, lactate dehydrogenase; CK, creatinine kinase; BUN, Blood urea nitrogen; sCr, serum creatinine; PT, prothrombin time; PTA, prothrombin activity; APTT, activated partial thromboplastin time; ALP, alkaline phosphatase; ALT, alanine aminotransferase; AST, aspartate aminotransferase; TB, total bilirubin; ALB, albumin; ADA, adenosine deaminase; UA, uric acid; CHE, choline esterase; NS, neurologic symptoms; NLR, neutrophil-to-lymphocyte ratio; PLR, platelet-to-lymphocyte ratio. 


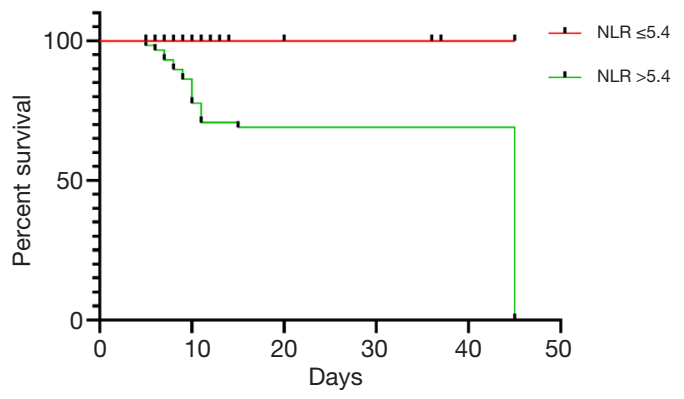

Figure 4 Survival curve of NLR. NLR, neutrophil-to-lymphocyte ratio.

infections in the acute phase; however, the overproduction or ineffective removal of NETs may cause tissue damage (29). Zhang et al. (30) tested the circulating cell-free DNA which could quantify the NETs content in the blood of SFTS patients and found that the concentration of circulating cell-free DNA in patients with severe SFTS was significantly higher than in patients with mild SFTS. Levels of cell-free DNA $(>711.7 \mathrm{ng} / \mathrm{mL})$ predicted severe illness in patients with SFTS $(\mathrm{OR}=8.285)$ (30). The results of several studies suggested that tumor-associated neutrophils (TANs) have various antitumor properties (31) and are capable of supporting tumor progression (32). This also suggested that we could further study the relationship between lymphocyte subsets and SFTS. The exact mechanism by which higher levels of NLR could affect the prognosis of the disease needs to be further examined.

Platelets and lymphocytes are derived from the same hematopoietic stem cells, and the PLR should be kept constant for homeostasis. However, platelets (10 days) and lymphocytes (several weeks to months) (33) have different lifespans, and in an abnormal state of hematopoiesis, the PLR might decrease because platelets are reduced faster than lymphocytes. Therefore, in theory, the PLR could be reduced in acute infections. Eren (18) suggested that in terms of viral hemorrhagic fever, the PLR might be used to predict the severity of the hemorrhagic fever. However, beyond our expectation, our study found that there was no difference in the PLR at the time of admission between the group that died and the group that survived. Moreover, there was no obvious correlation between the PLR and the risk model score, and Cox regression did not suggest that the PLR could be used as a risk factor for predicting SFTS death. We only found that the PLR was higher when the patients in the group that survived were discharged than when they were admitted, and it was statistically significant.
The corresponding mechanism is still unclear to us. We considered that one of the characteristics of this disease was thrombocytopenia, and the degree of thrombocytopenia was not significantly different between the group that died and the group that survived.

Several limitations of our study should be acknowledged. First, potential correlations between the PLR/NLR and anti-viral treatment outcomes were not investigated. Second, since we have not yet included the SFTSV viral nucleic acid load, we cannot clarify the link between SFTSV nucleic acid and the NLR and PLR. Third, we did not further divide the cohort into the severe group and the ordinary group or analyze the NLR and PLR regular changes and related risk prediction. Finally, due to the small number of deaths in our cohort, there may be statistical bias. Therefore, due to the limitations of this research, further studies are needed in the future.

\section{Conclusions}

SFTS is broadly distributed in central and northeastern China and the mortality rate is high. Our research shows that that is a difference between the NLR of the group that survives and the group that dies. Also, the NLR of patients in the recovery phase is lower than in the acute phase, which is consistent with the patient's improvement. An NLR greater than 5.4 can increase the risk of death and lessen the survival time of patients. However, the PLR has not yet shown a corresponding effect on the disease. In summary, the NLR provided a supplementary means for effectively managing SFTS.

\section{Acknowledgments}

The authors thank AiMi Academic Services (www. aimieditor.com) for English language editing and review services.

Funding: This study is supported by National Science and Technology Major Project of China (2018ZX09711003).

\section{Footnote}

Reporting Checklist: The authors have completed the STROBE reporting checklist. Available at http://dx.doi. org/10.21037/atm-20-4736

Data Sharing Statement: Available at http://dx.doi. org/10.21037/atm-20-4736 
Conflicts of Interest: All authors have completed the ICMJE uniform disclosure form (available at http://dx.doi. org/10.21037/atm-20-4736). The authors have no conflicts of interest to declare.

Ethical Statement: The authors are accountable for all aspects of the work in ensuring that questions related to the accuracy or integrity of any part of the work are appropriately investigated and resolved. The study was conducted in accordance with the Declaration of Helsinki (as revised in 2013) and the Harmonized Tripartite Guideline for Good Clinical Practice from the International Conference on Harmonization. This study was reviewed and approved by the Hamilton Integrated Research Ethics Board (approval number No. 2014-003) and the HoffmannLa Roche global review committee. All patients enrolled completed the informed consent form.

Open Access Statement: This is an Open Access article distributed in accordance with the Creative Commons Attribution-NonCommercial-NoDerivs 4.0 International License (CC BY-NC-ND 4.0), which permits the noncommercial replication and distribution of the article with the strict proviso that no changes or edits are made and the original work is properly cited (including links to both the formal publication through the relevant DOI and the license). See: https://creativecommons.org/licenses/by-nc-nd/4.0/.

\section{References}

1. Zhan J, Wang Q, Cheng J, et al. Current status of severe fever with thrombocytopenia syndrome in China. Virol Sin 2017;32:51-62.

2. Abudurexiti A, Adkins S, Alioto D, et al. Taxonomy of the order Bunyavirales: update 2019. Arch Virol 2019;164:1949-65.

3. Chen C, Li P, Li K, et al. Animals as amplification hosts in the spread of severe fever with thrombocytopenia syndrome virus: A systematic review and meta-analysis. Int J Infect Dis 2019;79:77-84.

4. Jiang XL, Zhang S, Jiang M, et al. A cluster of person-toperson transmission cases caused by SFTS virus in Penglai, China. Clin Microbiol Infect 2015;21:274-9.

5. Hu J, Shi C, Li Z, et al. A cluster of cases of severe fever with thrombocytopenia syndrome bunyavirus infection in China, 1996: A retrospective serological study. PLoS Negl Trop Dis 2018;12:e0006603.

6. Kim WY, Choi W, Park S, et al. Nosocomial Transmission of Severe Fever With Thrombocytopenia Syndrome in Korea. Clin Infect Dis 2015;60:1681-3.

7. Koga S, Takazono T, Ando T, et al. Severe Fever with Thrombocytopenia Syndrome Virus RNA in Semen, Japan. Emerg Infect Dis 2019;25:2127-8.

8. Mcmullan LK, Folk SM, Kelly A, et al. A New Phlebovirus Associated with Severe Febrile Illness in Missouri. N Engl J Med 2012;367:834-41.

9. Yu XJ, Liang MF, Zhang SY, et al. Fever with Thrombocytopenia Associated with a Novel Bunyavirus in China. N Engl J Med 2011;364:1523-32.

10. Li XK, Lu Q, Chen WW, et al. Arginine deficiency is involved in thrombocytopenia and immunosuppression in severe fever with thrombocytopenia syndrome. Sci Transl Med 2018;10:eaat4162.

11. Park SJ, Kim YI, Park A, et al. Ferret animal model of severe fever with thrombocytopenia syndrome phlebovirus for human lethal infection and pathogenesis. Nat Microbiol 2019;4:438-46.

12. Kim S, Eliot M, Koestler DC, et al. Association of Neutrophil-to-Lymphocyte Ratio with Mortality and Cardiovascular Disease in the Jackson Heart Study and Modification by the Duffy Antigen Variant. JAMA Cardiol 2018;3:455-62.

13. Eren SH, Zengin S, Buyuktuna SA, et al. Clinical severity in forecasting platelet to lymphocyte ratio in CrimeanCongo hemorrhagic fever patients. J Med Microbiol 2016;65:1100-4.

14. Verdoia M, Schaffer A, Barbieri L, et al. Impact of diabetes on neutrophil-to-lymphocyte ratio and its relationship to coronary artery disease. Diabetes Metab 2015;41:304-11.

15. Zitvogel L, Kepp O, Galluzzi L, et al. Inflammasomes in carcinogenesis and anticancer immune responses. Nat Immunol 2012;13:343-51.

16. Shaul ME, Fridlender ZG. Tumour-associated neutrophils in patients with cancer. Nat Rev Clin Oncol 2019;16:601-20.

17. Imamura M, Morimoto T, Egawa C, et al. Significance of baseline neutrophil-to-lymphocyte ratio for progressionfree survival of patients with HER2-positive breast cancer treated with trastuzumab emtansine. Sci Rep 2019;9:1811-10.

18. Wu W, Yan H, Zhao H, et al. Characteristics of systemic inflammation in hepatitis B-precipitated ACLF: Differentiate it from No-ACLF. Liver Int 2018;38:248-57.

19. Wang L, Wan G, Shen $Y$, et al. A nomogram to predict mortality in patients with severe fever with thrombocytopenia syndrome at the early stage- 
A multicenter study in China. PLoS Negl Trop Dis 2019; 13:e0007829.

20. Li H, Lu Q, Xing B, et al. Epidemiological and clinical features of laboratory-diagnosed severe fever with thrombocytopenia syndrome in China, 2011-17: a prospective observational study. Lancet Infect Dis 2018;18:1127-37.

21. Chen Y, Jia B, Liu Y, et al. Risk factors associated with fatality of severe fever with thrombocytopenia syndrome: a meta-analysis. Oncotarget 2017;8:89119-29.

22. Karasuyama H, Miyake K, Yoshikawa S, et al. Multifaceted roles of basophils in health and disease. J Allergy Clin Immunol 2018;142:370-80.

23. Yoshikawa S, Ohhora M, Hashimoto R, et al. Pivotal role of STIM2, but not STIM1, in IL-4 production by IL-3stimulated murine basophils. Sci Signa 2019;12:eaav2060.

24. Dellaquila E, Cremolini C, Zeppola T, et al. Prognostic and predictive role of neutrophil/lymphocytes ratio in metastatic colorectal cancer: a retrospective analysis of the TRIBE study by GONO. Ann Oncol 2018;29:924-30.

25. Zhao Z, Liu J, Wang J, et al. Platelet-to-lymphocyte ratio (PLR) and neutrophil-to-lymphocyte ratio (NLR) are associated with chronic hepatitis $\mathrm{B}$ virus (HBV) infection. Int Immunopharmacol 2017;51:1-8.

26. Liu H, Zhang H, Wan G, et al. Neutrophil-lymphocyte ratio: a novel predictor for short-term prognosis in acute-on-chronic hepatitis B liver failure. J Viral Hepat

Cite this article as: Wang $\mathrm{X}$, Lin L, Zhao Z, Zhou W, Ge Z, Shen Y, Wang L, Zhang W, Song R, Tian D, Wen J, Cui S, Yu X, Feng Y, Liu Y, Qiang C, Duan J, Ma Y, Li X, Fan T, Zhao Y, Chen Z. The predictive effect of the platelet-tolymphocyte ratio (PLR) and the neutrophil-to-lymphocyte ratio (NLR) on the risk of death in patients with severe fever with thrombocytopenia syndrome (SFTS): a multi-center study in China. Ann Transl Med 2021;9(3):208. doi: 10.21037/atm-204736
2014;21:499-507.

27. Templeton AJ, Knox JJ, Lin X, et al. Change in Neutrophil-to-lymphocyte Ratio in Response to Targeted Therapy for Metastatic Renal Cell Carcinoma as a Prognosticator and Biomarker of Efficacy. Eur Urol 2016;70:358-64.

28. Jenne CN, Wong CHY, Zemp FJ, et al. Neutrophils Recruited to Sites of Infection Protect from Virus Challenge by Releasing Neutrophil Extracellular Traps. Cell Host Microbe 2013;13:169-80.

29. Sorensen OE, Borregaard N. Neutrophil extracellular traps - the dark side of neutrophils. J Clin Invest 2016;126:1612-20.

30. Zhang Y, Song R, Shen Y, et al. High Levels of Circulating Cell-free DNA Are Associated With a Poor Prognosis in Patients With Severe Fever With Thrombocytopenia Syndrome. Clin Infect Dis 2020;70:1941-9.

31. Granot Z, Henke E, Comen EA, et al. Tumor Entrained Neutrophils Inhibit Seeding in the Premetastatic Lung. Cancer Cell 2011;20:300-14.

32. Galdiero MR, Varricchi G, Loffredo S, et al. Roles of neutrophils in cancer growth and progression. J Leukoc Biol 2018;103:457-64.

33. Harker LA, Roskos L, Marzec UM, et al. Effects of megakaryocyte growth and development factor on platelet production, platelet life span, and platelet function in healthy human volunteers. Blood 2000;95:2514-22. 